\title{
一种基于多尺度卷积神经网络和分类统计的图像去雾䨪方法
}

\author{
齐永锋, 李占华 \\ (西北师范大学 计算机科学与工程学院, 甘肃 兰州 730070)
}

\begin{abstract}
摘要：传统的去雾皬方法会导致天空、白云和明亮区域内的颜色失真。为了解决以上问题, 提出了一 种基于多尺度卷积神经网络和分类统计的去除图像雾皬的方法。首先用多尺度卷积神经网络估计图像 的透射率，其次对所估计的透射率进行分类统计以确定在暗通道内天空、白云和明亮区域的像素值， 最后通过低通高斯滤波器平滑图像场景的辐射度, 得到恢复的无雾霔图像。实验结果表明, 采用提出 的方法对图像去雾䨪后明亮区域内的颜色不会失真，且保留了图像的自然外观，对合成图像和真实图 像均有较好的去雾皬效果。
\end{abstract}

关键词：图像处理；图像去雾霧；多尺度卷积神经网络；分类统计；透射率

中图分类号：TN219 文献标识码：A 文章编号：1001-8891(2020)02-0190-08

\section{Image Dehazing Method Based on Multi-scale Convolutional Neural Network and Classification Statistics}

\author{
QI Yongfeng, LI Zhanhua
}

(School of Computer Science and Engineering, Northwest Normal University, Lanzhou 730070, China)

\begin{abstract}
Traditional methods of image dehazing can distort color in areas such as the sky, white clouds, and bright areas. To address these problems, a three-step method is proposed for removing image hazing using a multi-scale convolutional neural network (MCNN) and classification statistics. First, the MCNN is used to estimate the transmittance of the image. Second, the estimated transmittance is classified and the pixel values of the sky, white clouds, and other bright regions in the dark channel are determined. Finally, the radiance of the scene is smoothed by a low-pass Gaussian filter to produce a restored haze-free image. Experimental results show that this method preserves the color in bright areas after the image is defogged, retaining the natural appearance of the image. The proposed method achieves improved dehazing on both synthetic and real images.
\end{abstract}

Key words: image processing, image dehazing, multi-scale convolutional neural network, classification statistics, transmittance

\section{0 引言}

雾靁中的悬浮颗粒具有散射和吸收的作用, 使得 室外图像呈现出较低的对比度和有限的可见性, 从而 导致在雾䨪天采集到的图像质量较低, 限制了图像在 智能识别、目标监控、目标追踪等方面的应用。为使 计算机系统能更好地识别和提取相关的图像特征, 去 雾䨪技术成为计算机视觉领域的研究热点 ${ }^{[1-5]}$ 。

目前图像去雾䨪的方法大致分为两种：基于自 适应对比度增强的方法 ${ }^{[6-7]}$ 和基于透射率估计的方
法 ${ }^{[8-13]}$ 。前一种方法又分为全局增强的方法和局部化 增强的方法, 其中全局化增强的方法包括全局直方图 均衡化 ${ }^{[14]} 、$ Retinex ${ }^{[15]}$ 等。而局部化增强的方法包括局 部直方图均衡化 ${ }^{[16]}$ 、局部对比度增强方法 ${ }^{[17]}$ 等。自适 应对比度增强的方法虽然可以排除图像中的部分干 扰信息, 但未对雾霧天图像的成像原理进行分析, 只 是通过增强对比度去除雾䨚, 因而对雾霉严重的区域 无法特殊处理。透射率估计的方法依据大气散射模 型, 从光学成像的本质上去除雾霧, 通过图像退化基 理和先验知识恢复无雾䨪图像, 去雾䨪效果较好, 因 
而得到了广泛的研究和应用。根据图像先验知识估计 大气透射率, 例如暗通道先验 (dark channel prior,

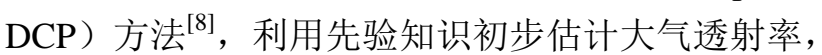
再用软抠图细化透射率, 最终根据大气光物理散射模 型恢复无雾䨪的图像。该方法假设在清晰图像中的暗 通道值接近于零, 然而当场景中的物体与大气光相似 时该假设并不成立, 同时由于软抠图计算复杂, 因此 该算法复杂度高、耗时大。

基于大气散射模型的方法由于估计透射率成本 较高, Tang 等人 ${ }^{[18]}$ 将机器学习算法应用到去雾雽算 法中, 以各种先验特征为输入, 通过随机森林模型估 计透射率, 从而降低透射率的估计成本, 但该方法对 雾度分布不均匀的图像去雾霌效果并不是很理想, 且 存在明亮区域内去除雾霧时颜色被过度增强的问题。 Meng 等人 ${ }^{[19]}$ 应用透射函数固有的边界约束, 将该约 束与加权 $\mathrm{L}_{1}$ 范数的上下文正则化相结合, 估计图像的 透射率, 该方法往往产生锐利的边缘和高度对比的颜 色。Cai 等人 ${ }^{[20]}$ 以卷积神经网络 (convolutional neural networks, CNN) 架构为基础, 提出可训练的端到端 系统进行透射率的估计, 根据大气散射模型从清晰图 像生成雾皬图像的数据库, 该方法通过大气散射模型 的逆运算计算清晰图像, 去雾雽效果会受到模型其它 参数的影响。

Ren 等人 ${ }^{[21]}$ 提出了多尺度卷积神经网络 (multi-scale convolutional neural network, MSCNN) 估计大气透射率, 该方法在真实雾䨪图像中的主观评 估结果较好, 但合成室内图像的峰值信噪比 (peak signal to noise ratio, PSNR) 和结构相似性 (structural similarity index, SSIM) 结果较低, 其在场景识别中 对天空、白云和明亮区域的识别性能也较低。针对以 上算法的不足, 本文提出了一种新的改进算法。在多 尺度卷积神经网络模型中将粗尺度网络估计的透射 率的输出作为附加特征图传递到精细尺度网络后, 通 过分类统计的方式确定明亮区域的像素值, 该统计方 法实现了像素的运算, 不仅减弱了阴影的影响而且避 免了明亮区域被过度增强的现象, 并进一步采用高斯 滤波器提高图像质量, 通过平滑所求的场景辐射度得 到最终无雾䨟图像。

\section{1 基本理论}

\section{1 大气散射模型}

图像去雾霔算法中广泛使用的退化模型是 McCartney 等人 ${ }^{[22]}$ 提出的大气散射模型, 其表达如式 (1)所示:

$$
I(x)=J(x) t(x)+A[1-t(x)]
$$

式中: $I(x)$ 和 $J(x)$ 分别表示为观察到的雾䨪图像和无雾 䨪图像的场景辐射度; $A$ 表示全球大气光; $t(x)$ 是场景 的透射率, 即光线透过媒介透射到视觉系统没有被散 射的部分, $t(x)$ 表示为:

$$
t(x)=\mathrm{e}^{-\beta d(x)}
$$

式中: $\beta$ 是大气中的介质消光系数; $d(x)$ 是场景深度。 由于给定的雾霻图像存在多种解决方案, 显然这个问 题是病态的。若能直接得到图像大气透射率 $t(x)$ 和全 球大气光 $A$, 那么在雾䨚图像已知的情况下, 根据式 (1)恢复原始无雾靁图像。为获得大气透射率 $t(x)$, 本 文结合多尺度卷积神经网络和分类统计的方法估计 大气透射率 $t(x)$, 先用多尺度卷积神经网络进行 $t(x)$ 值的初步计算, 再对所计算的 $t(x)$ 值进行分类统计。

\section{2 多尺度卷积神经网络的模型体系结构}

为实现图像透射率 $t(x)$ 的初步估计, 采用了多尺 度卷积神经网络模型 ${ }^{[23]}$, 该模型分为 2 部分: 粗尺度 网络和精细尺度网络。

多尺度卷积神经网络模型如图 1 所示。一个粗尺 度的网络首先对透射率进行整体的估计, 然后通过一 个精细的网络在局部区域内对其进行修改。这两个组 成部分都运用原始雾霳图像作为输入。此外, 粗尺度 网络的输出作为附加特征图传递到精细尺度网络, 通 过这种方式, 精细尺度网络能够细化粗尺度网络。

\subsection{1 粗尺度网络}

粗尺度网络的目标是用场景的全局视图预测图 像的整体透射率, 粗尺度网络由 4 部分组成: 卷积, 最大池化, 上采样, 线性组合。

卷积: 该模型将 RGB 图像作为输入, 卷积层由 与输入特征图卷积的滤波器组组成, 每个卷积层表示 为:

$$
f_{n}^{l+1}=\sigma\left[\sum_{m}\left(f_{m}{ }^{l} * k_{m, n}{ }^{l+1}\right)+b_{n}{ }^{l+1}\right]
$$

式中: $f_{m}{ }^{l}$ 和 $f_{n}{ }^{l+1}$ 分别表示为当前层 $l$ 的特征图到下一 层 $l+1$ 的特征图; $k$ 表示卷积核, 索引 $(m, n)$ 表示从当 前层第 $m$ 个特征映射到下一层第 $n$ 个特征的映射; 表示卷积; 函数 $\sigma(\cdot)$ 表示滤波器上的修正线性单元, 所有隐藏层都使用修正线性单元进行激活; $b$ 表示的 是偏置单元。

最大池化: 在每个卷积层之后使用降采样因子为 2 的最大池化层, 保留了更多的纹理信息。

上采样: 在卷积中由于输入雾霻图像与输出透射 率的大小是一样的, 而特征图的大小在最大池化层之 后将会减小。因此, 在最大池化层后增加了一个上采 样层 ${ }^{[24]}$, 以确保输入雾雽图像和输出透射率的大小相 等。上采样层紧随池化层之后将恢复子采样特征的大 小，同时保持网络的非线性。每个上采样层被定义为: 


$$
f_{n}^{l+1}(2 x-1: 2 x, 2 y-1: 2 y)=f_{n}^{l}(x, y)
$$

该函数将位置 $(x, y)$ 处的像素值从最大池化特征复 制到紧随其后的上采样层中大小为 $2 \times 2$ 的块上, 因 为上采样层中的每个块由同样大小的值组成, 这个层 的反向传播规则就是反向的平均池化层, 其尺度因子 为 2 。每个上采样层中 $2 \times 2$ 的块函数被定义为:

$$
f_{n}^{l}(x, y)=\frac{1}{4} \sum_{2 \times 2 f_{n}^{l+1}}(2 x-1: 2 x, 2 y-1: 2 y)
$$

线性组合: 在粗尺度卷积网络中输出层通过线性 组合 ${ }^{[23]}$ 将来自最后一个卷积块的特征通道组合在一 起。然后用 sigmoid 激活函数产生最终输出。sigmoid 函数表示为:

$$
f(x)=1 /\left(1+\mathrm{e}^{-x}\right)
$$

用 sigmoid 激活函数产生最终的输出:

$$
t_{\mathrm{c}}=s\left(\sum_{n} \omega_{n} f_{n}^{p}+b\right)
$$

式中: $t_{\mathrm{c}}$ 在粗尺度网络中表示场景透射率的输出; $n$ 是特征映射通道的索引; $s(\cdot)$ 是 sigmoid 激活函数; $f_{n}{ }^{p}$ 表示输出透射率前的倒数第二个特征映射; $\omega$ 和 $b$ 分 别是线性组合的权重和偏置单元。

$$
t(x)= \begin{cases}1-\omega \min _{c \in\{R, G, B\}}\left(\min _{x \in \Omega(x)}\left(\frac{I^{c}(x)}{A}\right)\right) & , I^{\mathrm{dark}}(x)<160 \\ 1.035^{I^{\mathrm{dark}}(x)-160} *\left(1-\omega \min _{c \in\{R, G, B\}}\left(\min _{x \in \Omega(x)}\left(\frac{I^{c}(x)}{A}\right)\right)\right), I^{\mathrm{dark}}(x)>=160\end{cases}
$$

式中： $\omega$ 取值为 0.75 。

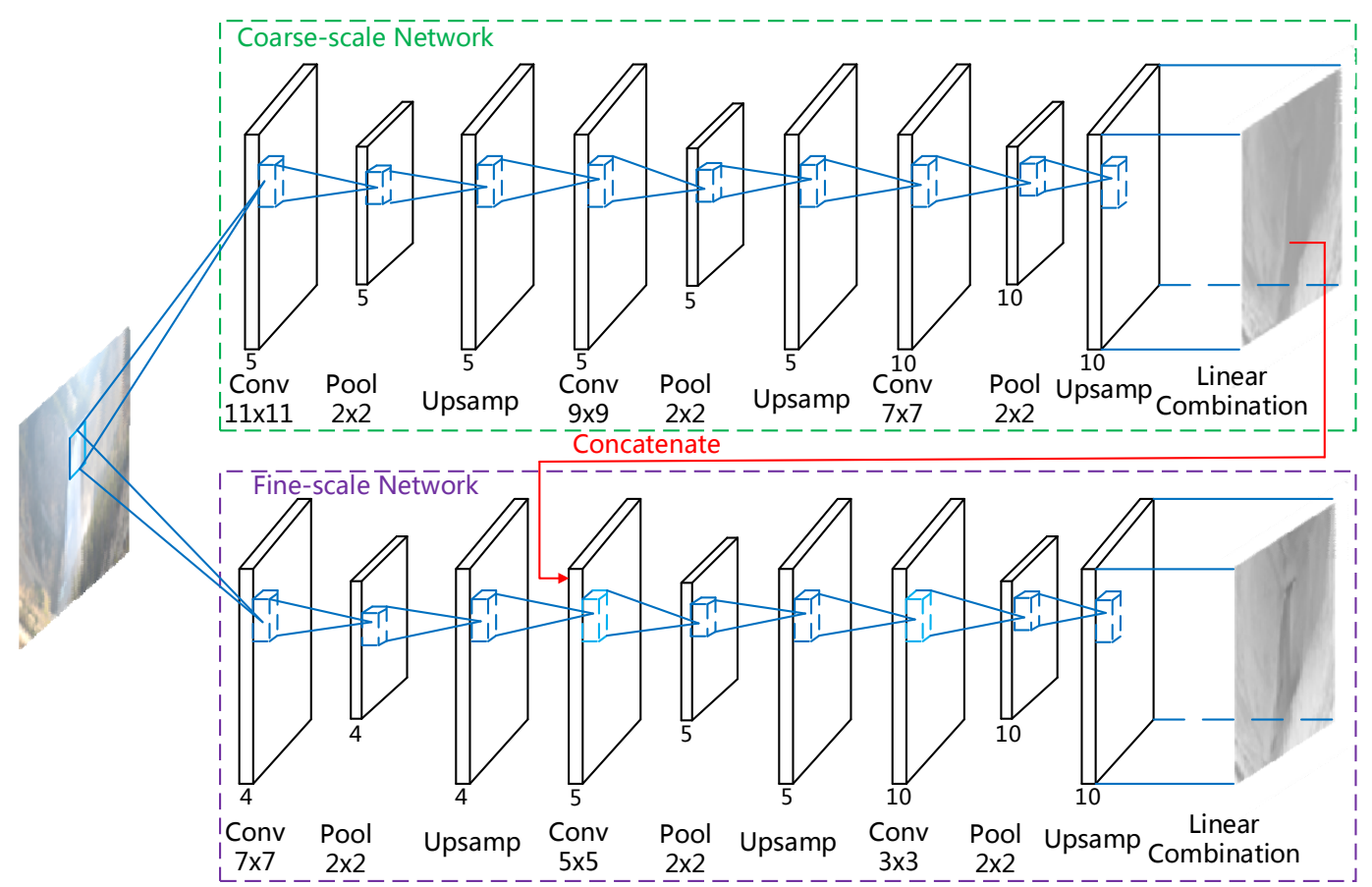

图 1 多尺度卷积神经网络模型

Fig.1 Model diagram of multi-scale convolution neural network 


\section{4 大气光的估计}

为了恢复清晰的图像除了需要估计透射率 $t(x)$ 外 还需要估计大气光 $A$, 由(1)式可知, 当 $t(x) \rightarrow 0$ 时 $I(x)$ $=A$ 。由于在室外图像中出现的物体可能远离观察者, 所以深度 $d(x)$ 的范围为 $[0,+\infty)$, 并且当 $d(x) \rightarrow \infty$ 时有 $t(x)=0$ 。因此在透射率 $t(x)$ 中通过选择 $0.1 \%$ 暗像素来 估计大气光 $A$ 。现已得到图像大气透射率 $t(x)$ 和全球 大气光 $A$, 那么在雾雺图像已知的情况下, 根据式(1) 恢复原始无雾霳图像, 得到无雾䨪图像场景的辐射度 $J(x)$ 。

\section{5 无雾䨪图像场景的辐射度}

在估计 $t(x)$ 和 $A$ 后, 用式(1)恢复无雾雱图像, 为 了避免更多噪声的影响, 对 $t$ 设置一个下限 $t_{0}$ 为 0.1 , 这样处理后图像较自然, 因此最终的无雾䨪图像场景 的辐射度为:

$$
J(x)=\frac{I(x)-A}{\max \{t(x), 0.1\}}+A
$$

得到无雾图像场景的辐射度 $J(x)$ 后, 然后采用高 斯滤波器进行平滑处理。

\section{6 高斯滤波平滑无雾霾图像场景的辐射度}

利用低通高斯滤波器平滑上述得到的无雾霳图 像场景的辐射度, 精化辐射轮廓 $V(x)$ 表示为:

$$
V(x)=\frac{1}{W^{g}} \sum_{y \in s} G_{\sigma}(\|x-y\|) \bar{V}(y)
$$

式中: $W^{g}$ 是以像素 $x$ 为中心的邻域区块的整体权重。

$$
W^{g}=G_{\sigma}(\|x-y\|)
$$

式中: $G$ 是高斯函数, 并且参数 $\sigma$ 表示用于平滑像素 的区域的大小, 高斯传播的标准偏差确定为标量或正 值的 3 分量矢量。根据低通高斯滤波器精化场景辐射 度, 使靠近聚焦像素 $x$ 的像素将获得相当大的权重。

图像去雾䨪算法流程如下所示:

输入: 雾䨪图像 $I(x)$

输出: 无雾霧图像场景的辐射度 $J(x)$

Step 1: 用 $11 \times 11 \times 5$ 的三维卷积核对原始输入 层进行卷积, 通过最大池化和上采样实现色差、纹理 和对比度特征的提取, 输出雾霳图像的整体透射率 $t(x)$ 。

Step 2: 将 Step 1 的输出作为附加特征图传递给 精细网络进行细化, 估计透射率 $t(x)$ 。

Step 3: 分类统计特定区域内雾皬图像的大气透 射率 $t(x)$ 。

$$
\begin{aligned}
& \text { If } I^{\mathrm{dark}}(x)<160 \\
& t(x)=1-\omega * I^{\mathrm{dark}}(x) . / \mathrm{A}
\end{aligned}
$$

Else

$$
t(x)=1.035^{\wedge}\left(I^{\mathrm{dark}}(x)-160\right) *\left[1-\omega * I^{\mathrm{dark}}(x) . / A\right]
$$

Step 4: 估计全球大气光 $A$ 。

Step 5: 根据式(1)得无雾霳图像的场景辐射度 $J(x)$ $=[I(x)-A] / \max [t(x), 0.1]+A$ 。

Step 6: 用高斯滤波器平滑 Step 5 所得的结果 $J(x)$ 。

\section{2 实验}

\section{1 实验数据}

训练集: 通常情况下不可能捕捉到同样视觉场景 下有雾霧和无雾䨪而其它所有环境条件都相同的的 图像。所以只能用无雾雽图像合成雾䨪图像得到训练 集。用公开的 indoor training set (ITS) 和 outdoor training set (OTS) ${ }^{[26]}$ 数据集, ITS 室内合成训练集中 包含 110000 张图片, OTS 室外合成训练集中包含 313950 张图片。为了控制训练的数据量同时保证训练 模型的泛化能力, 本文分别从 ITS 和 OTS 中选取 1100 张和 3100 张图片组成混合训练集。为了适应多尺度 网络模型的尺度, 所有的训练图像都被调整为 $320 \times 240$ 像素的标准尺寸。

测试集: 为了验证本文模型的实际性能表现, 从 synthetic objective testing set (SOTS) 和 hybrid subjective testing set (HSTS) 数据集 ${ }^{[26]}$ 的 50 幅图像 生成雾天图像测试集。利用峰值信噪比和结构相似性 作为评价结果的量化标准。

\section{2 实验设置}

实验中粗尺度网络和精细网络均由 3 个卷积层构 成, 在粗尺度网络中前两层分别用了 5 个大小为 $11 \times 11$ 和 $9 \times 9$ 的卷积层, 最后一层是由大小为 $7 \times 7$ 的 10 个滤波器组成。在精细网络中, 第一个卷积层 是由大小为 $7 \times 7$ 的 4 个滤波器组成, 最后两层分别 由大小为 $5 \times 5$ 的 5 个滤波器和 $3 \times 3$ 的 10 个滤波器 组成, 粗尺度网络和精细尺度网络都是用具有 0.9 动 量的随机梯度下降法训练, 实验使用了 100 幅图像 (320 $\times 240$ 个像素) 的批量大小, 初始学习速率设置 为 0.001 , 每迭代 20 次初始学习速率为原来的 0.1 倍, 迭代次数设置为 70 次。如图 2(a)第一、第二和第三个 尺度网络之间的比较表示更大规模的网络不会带来 更好的效果。图 2(b)更多层的 CNN 和提出的多尺度 CNN 之间的比较表示多尺度 CNN 训练效果比多层的 CNN 好。

\section{3 实验结果与分析}

\section{1 定量分析}

为了更加客观地评价方法的有效性, 首先在合成 数据集上定量评估所提出的透射率估计性能的方法。 利用峰值信噪比和结构相似性将所提出的算法与其 
他的去雾䨪方法 ${ }^{[8,13,21]}$ 进行了比较。表 1 给出了本文 的方法和其他方法的比较结果。

\section{2 直观效果分析}

为了直观验证提出的方法去雾䨪的有效性, 分别 在室内合成图像和室外真实图像上对所提方法和目 前常用的去雾䨪方法进行直观效果分析对比。首先用 室内合成的雾霳图像比较各算法的去雾䨪效果。如图 3 所示, $\mathrm{DSPP}^{[13]}$ 的算法能够增加图像细节并增强图像 的可见性。但是, 恢复的图像中仍有颜色扭曲, 如第 2 幅图像的桌面部分。而 $\mathrm{DCP}^{[8]}$ 的方法高估了雾的厚 度并产生较暗的结果, 如第 3 幅图的桌面部分和第 4 幅图的地面部分。MSCNN ${ }^{[21]}$ 的方法总体去雾霧效果 较好, 但会显著增强图像明亮区域。相比之下, 图 3(f) 的去雾皬效果基本接近于真实无雾图像, 这表明本文 的方法去雾霧效果较好。

其次比较各算法在室外真实雾䨪场景中的效果。 图 4 中 $\mathrm{DSPP}^{[13]}$ 提出的方法存在图像丢失细节、过度 增强图像明亮区域的问题。如第 1、2 幅图的白云部 分。此外,第 3 幅中也增强了蓝天、白云的亮度。DCP ${ }^{[8]}$

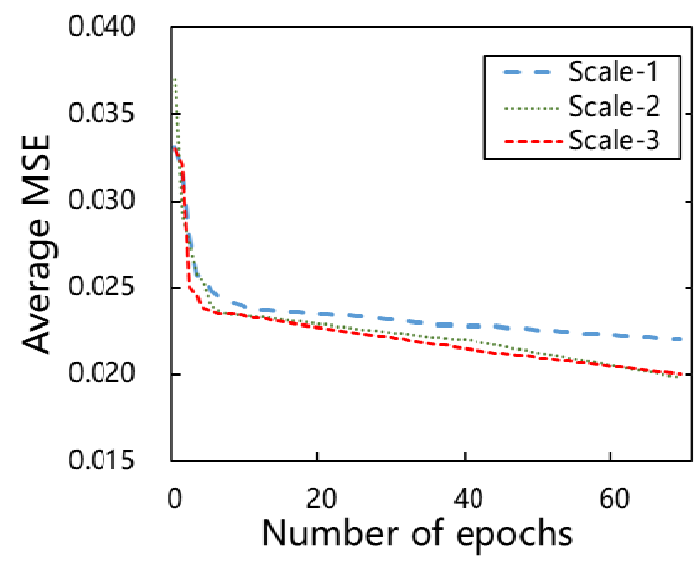

(a) 第一、第二和第三尺度网络之间的比较 (a) Comparisons among the first, second and third scale networks

图 2 各尺度网络以及多层网络之间的比较 Fig.2 Fig.2 Cor

去雾䨪效果较好, 但颜色仍然比实际情况更暗。相比 之下, $\mathrm{MSCNN}^{[21]}$ 的方法和本文所提出的方法在真实 雾霍场景中关于天空、白云和明亮区域的主观评估结 果差异不大, 因而本文将在 3.3 节用场景的识别做进 一步对比分析。

\section{3 场景识别中结果分析}

在 3.2 节室外真实雾雮场景直观效果分析中 MSCNN 算法和本文算法直观效果难以区分。为了进 一步证明本文提出的算法去雾霔结果在场景识别中 的性能, 用 Google Vision API ( https://cloud. google.com/vision）测试真实雾霛图像增强之后是否 提高场景识别能力。对比 MSCNN 和本文算法在场景 识别性能上的表现, 结果如图 5 所示。可以看出, 本 文算法在雾䨪图像增强之后的场景识别中比 MSCNN 算法识别效果更好, 尤其是在天空、白云和明亮区域 表现更佳。如图 5 Google Vision API 识别结果示例中 显示, 本文方法在雾霧图像增强之后会增加主要对象 检测的分数。如(c)中天空和白云的分数较(a)和(b)都 有较大提升。

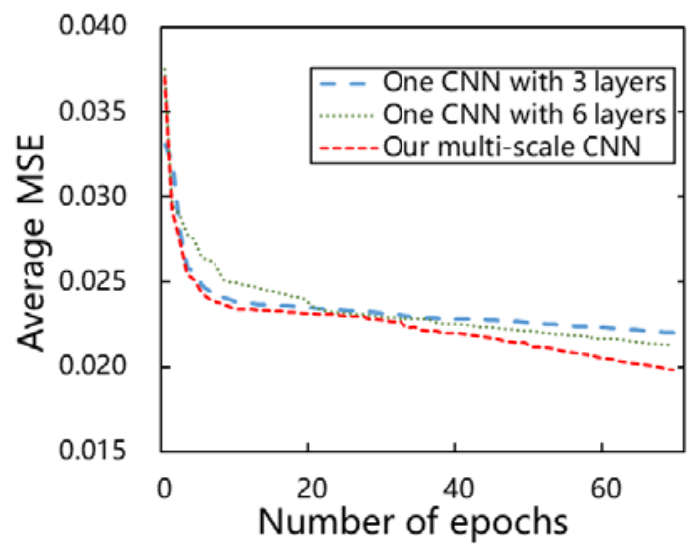

(b) 多层的 CNN 和多尺度 CNN 之间的比较 (b) Comparisons of one CNN with more layers and the multi-scale CNN

Comparisons between scale networks and multi-layer networks 表 1 合成数据集的平均 PSNR 和 SSIM Table 1 Average PSNR and SSIM of the synthesized data set

\begin{tabular}{cccccc}
\hline & Image & MSCNN $^{[21]}$ & DCP $^{[8]}$ & DSPP $^{[13]}$ & Ours \\
\hline \multirow{5}{*}{ Indoor } & 1400 & $18.12 / 0.78$ & $16.17 / 0.69$ & $11.17 / 0.79$ & $19.47 / 0.95$ \\
& 1401 & $18.27 / 0.86$ & $17.51 / 0.71$ & $13.21 / 0.72$ & $20.07 / 0.93$ \\
& 1402 & $19.84 / 0.90$ & $19.45 / 0.55$ & $17.61 / 0.83$ & $20.39 / 0.89$ \\
& 1403 & $18.29 / 0.86$ & $17.39 / 0.80$ & $16,57 / 0.73$ & $19.84 / 0.91$ \\
& 1404 & $18.87 / 0.85$ & $19.53 / 0.77$ & $12.85 / 0.69$ & $20.47 / 0.89$ \\
& 0001 & $19.56 / 0.83$ & $17.67 / 0.64$ & $10.39 / 0.55$ & $21.01 / 0.96$ \\
\multirow{3}{*}{ Outdoor } & 0002 & $18.11 / 0.88$ & $18.29 / 0.75$ & $11.30 / 0.62$ & $21.15 / 0.94$ \\
& 0003 & $17.08 / 0.76$ & $16.54 / 0.63$ & $11.87 / 0.66$ & $21.21 / 0.95$ \\
& 0004 & $18.83 / 0.92$ & $16.88 / 0.56$ & $15.89 / 0.78$ & $19.35 / 0.93$ \\
& 0005 & $19.35 / 0.88$ & $17.33 / 0.58$ & $13.93 / 0.73$ & $19.88 / 0.94$ \\
& Average & $18.63 / 0.85$ & $17.68 / 0.67$ & $13.50 / 0.71$ & $20.30 / 0.93$ \\
\hline
\end{tabular}




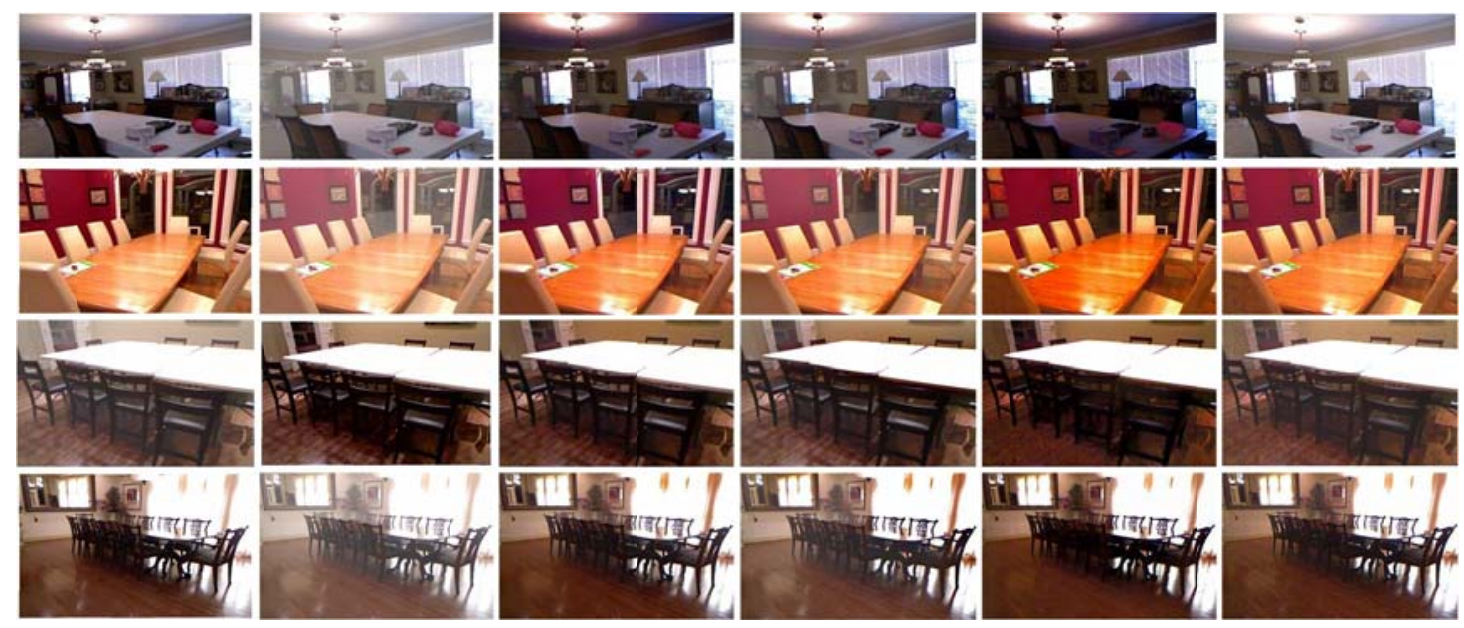
(a) Origin
(b) Inputs
(c) MSCNN
(d) DCP
(e) DSPP
(f) Ours

图 3 室内合成图像去雾霳结果对比 Fig.3 Comparisons of indoor synthetic images dehazy results

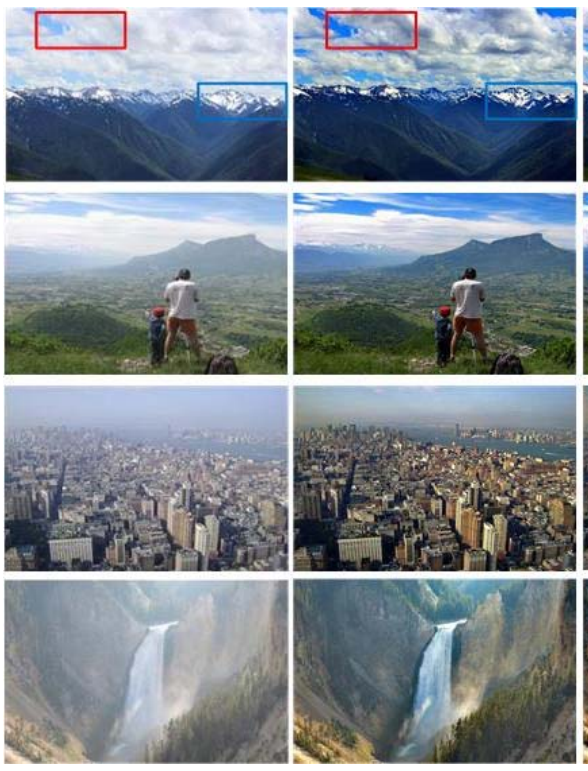

(a) Inputs

(b) MSCNN
图 4 真实图像去雾䨪结果对比
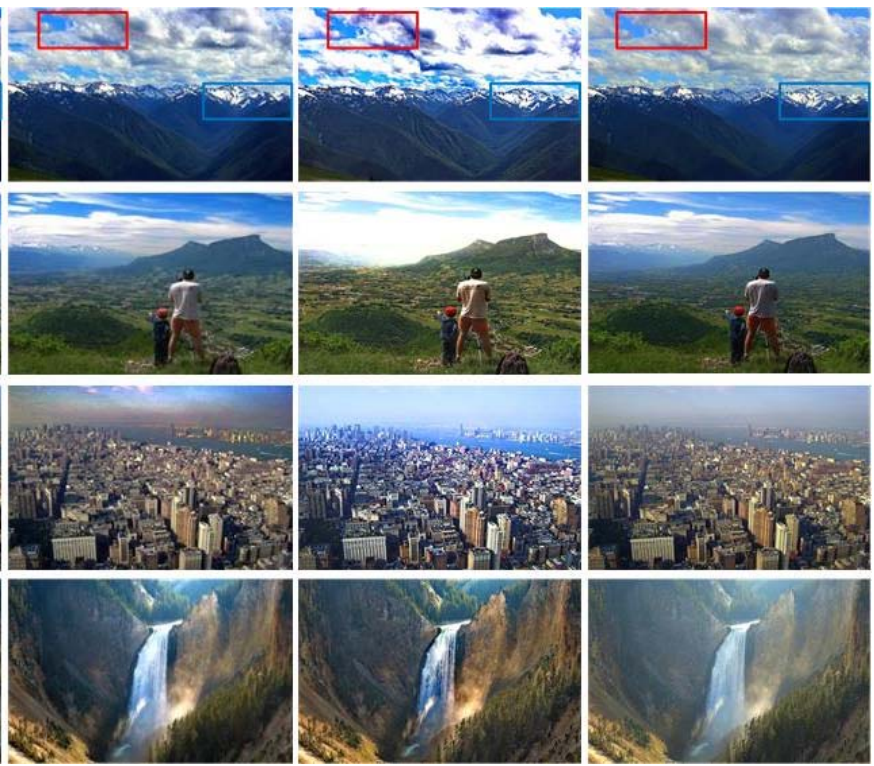

(c) DCP

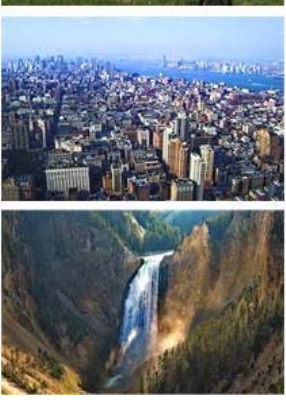

(d) DSPP

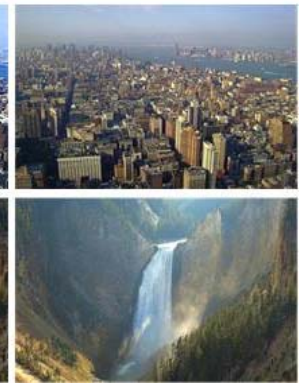

(e) Ours

Fig.4 Comparisons of real image dehazy results

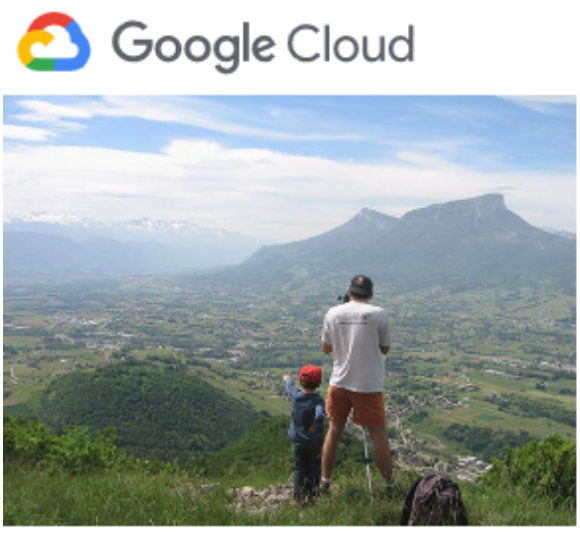

\begin{tabular}{lc} 
Ridge & $94 \%$ \\
\hline Sky & $86 \%$ \\
\hline Hill Station & $89 \%$ \\
\hline Cloud & $60 \%$ \\
\hline Valley & $76 \%$ \\
\hline Grass & $57 \%$ \\
\hline
\end{tabular}

(a) 原始雾䨪图像识别结果

(a) Original haze image recognition result 


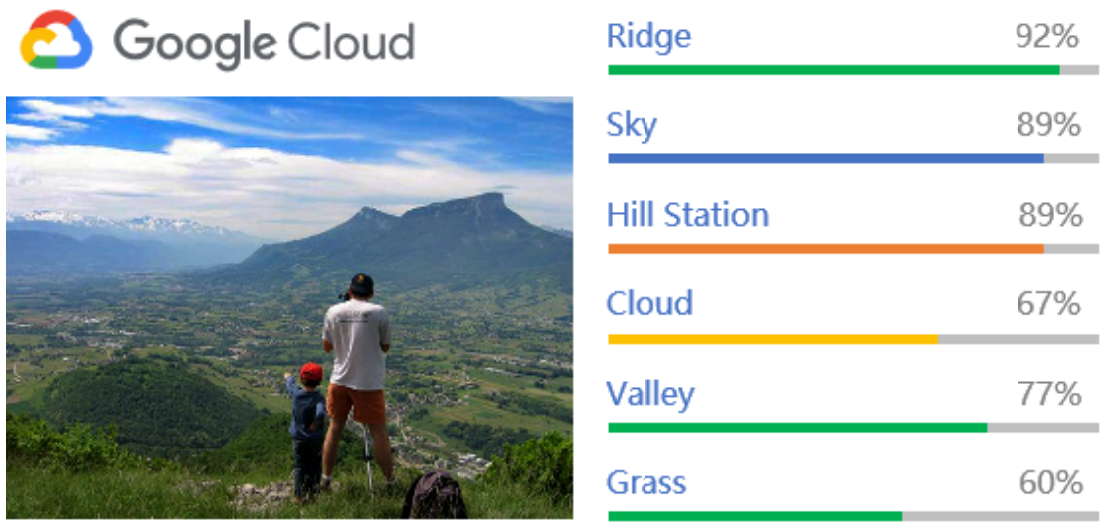

(b) MSCNN 算法去雾䨪后识别结果(b) MSCNN algorithm after dehazy recognition results

\begin{tabular}{lll}
3 Google Cloud & \multicolumn{1}{l}{ Ridge } & $94 \%$ \\
\cline { 2 - 3 } & Sky & $91 \%$ \\
\hline & Hill Station & $96 \%$ \\
\hline & Cloud & $78 \%$ \\
\hline & Valley & $77 \%$ \\
\hline
\end{tabular}

(c) 本文算法去雾霾后识别结果(c) Identification results of the algorithm after hazy removal

图 5 MSCNN 算法和本文算法识别结果对比

Fig.5 Comparisons between MSCNN algorithm and the recognition result of the algorithm

\section{4 结论}

本文结合多尺度卷积神经网络和分类统计的方 法改善原有算法在透射率计算上的问题, 通过调整算 法中参数并且给出相应参数的合适范围, 确保最终的 去雾皬效果。通过定量和直观效果分析的结果对比, 本文算法能够有效地解决特定区域内颜色失真、颜色 扭曲及过度增强等问题, 且在合成的雾皬图像和真实 的雾霧图像中都有较好的效果。在接下来的研究中, 如何提高去雾霳图像的分辨率将是一个重点, 以期进 一步提高去雾䨪图像的分辨率。

\section{参考文献:}

[1] 李明, 杨艳屏. TV-Retinex: 一种快速图像增强算法[J]. 计算机辅助设 计与图形学学报, 2010, 22(10): 1778-1782.

LI M, YANG Y P. TV-Retinex: a fast image enhancement algorithm[J]. Journal of Computer-Aided Design and Computer Graphics, 2010, 22(10): 1778-1782.

[2] 郭璠, 蔡自兴. 图像去雾算法清晰化效果客观评价方法 $[\mathrm{J}]$. 自动化学 报, 2012, 38(9): 1410-1419.

GUO F, CAI Z X. Image removal of haze algorithm clear effect objective evaluation method[J]. Acta Automatica Sinica, 2012, 38(9): 1410-1419.
[3] Milanfar P, Matlin E. Removal of haze and noise from a single image[C]//Proceedings of SPIE, 2012, 8296(3): 17.

[4] DENG L, LI O X, ZHAO S W. An improved image defogging algorithm based on global dark channel prior and fuzzy logic control[C]//The 12th International Computer Conf. on Wavelet Active Media Technology and Information Processing, 2015: 188-191.

[5] 徐岩, 孙美双. 基于多特征融合的卷积神经网络图像去雾算法 [J]. 激 光与光电子学进展, 2018, 55(3): 254-263.

XU Y, SUN M S. Convolution neural network image defogging based on multi feature fusion[J]. Laser \& Optoelectronics Progress, 2018, 55(3): 254-263.

[6] Caraa L, Tarel J P. Markov random field model for single image defogging[C]//IEEE Intelligent Vehicle Symposium, 2013: 994-999.

[7] LI Z, TAN P, ZOU D P, et al. Simultaneous video defogging and stereo reconstruction[C]//IEEE Conference on Computer Vision and Pattern Recognition (CVPR), 2015: 4988-4997.

[8] HE K, SUN J, TANG X. Single image haze removal using dark channel prior[J]. IEEE Transactions on Pattern Analysis and Machine Intelligence (TPAMI), 2011, 33(12): 2341-2353.

[9] 王多超, 王永国, 董雪梅, 等. 贝叶斯框架下的单幅图像去雾算法 [J]. 计算机辅助设计与图形学学报, 2010, 22(10): 1756-1761. 
WANG D C, WANG Y G, DONG X M, et al. Single image dehazing based on Bayesian framework[J]. Journal of Computer-Aided Design and Computer Graphics, 2010, 22(10): 1756-1761.

[10] Ancuti C O, Ancuti C. Single image dehazing by multiscale fusion[J]. IEEE Transactions on Image Processing, 2013, 22(8): 3271-3282.

[11] 鞠铭烨, 张登银, 纪应天. 基于雾气浓度估计的图像去雾算法[J]. 自 动化学报, 2016, 42(9): 1367-1379.

JU M Y, ZHANG D Y, JI Y T. Image haze removal algorithm based on haze thickness estimation[J]. Acta Automatica Sinica, 2016, 42(9): 1367-1379.

[12] CHEN C. DOM N, WANG J. Robust image and video dehazing with visual artifact suppression via gradient residual minimization[C]// Computer Vision - ECCV, 2016, 9906: 576-591.

[13] HE L, ZHAO J, ZHENG $\mathrm{N}$, et al. Haze removal using the difference-structure-preservation prior[J]. IEEE Transactions on Image Processing, 2017, 26(3): 1063-1075.

[14] Stark J A. Adaptive image contrast enhancement using generalizations of histogram equalization[J]. IEEE Transactions on Image Processing, 2000, 9(5): 889-896.

[15] YANG W, WANG R, FANG S, et al. Variable filter Retinex algorithm for foggy image enhancement[J]. Journal of Computer-Aided Design \& Computer Graphics, 2010, 22(6): 965-971.

[16] XU Z, LIU X, JI N. Fog removal from color images using contrast limited adaptive histogram equalization[C]//CISP'09 of IEEE, 2009: 1-5.

[17] TAN R. Visibility in bad weather from a single image[C]// Processing of IEEE Conference on Computer Vision and Pattern Recognition(CVPR), 2008: 2347-2354.
[18] TANG K, YANG J, WANG J. Investigating haze-relevant features in a learning framework for image dehazing[C]//Proceedings of the IEEE Conference on Computer Vision and Pattern Recognition, 2014: 2995-3000.

[19] MENG G, WANG Y, DUAN J, et al. Efficient image dehazing with boundary constraint and contextual regularization[C]//Proceedings of the IEEE international Conference on Computer Vision, 2013: 617-624.

[20] CAI B, XU X, JIA K, et al. Dehazenet: an end-to-end system for single image haze removal[J]. IEEE Transactions on Image Processing, 2016, 25(11): 5187-5198.

[21] REN W, LIU S, ZHANG H, et al. Single image dehazing via multi-scale convolutional neural networks[C]//European Conference on Computer Vision, 2016: 154-169.

[22] McCartney E J. Optics of the atmosphere-scattering by molecules and particles[J]. Optica Acta: International Journal of Optics, 1977, 30(5): 76.

[23] Sermanet P, LeCun Y. Traffic sign recognition with multi-scale convolutional networks[C]//Neural Networks(IJCNN) of IEEE Conference, 2011: 2809-2813.

[24] YUAN J, NI B, Kassim A. Half-CNN: a general framework for whole-image regression[J]. arXiv Preprint arXiv, 2014: 1412.6885.

[25] HUANG C, YANG D, ZHANG R, et al. Improved algorithm for image haze removal based on dark channel priority[J]. Computers \& Electrical Engineering, 2017:(DOI: 10.1016/j.compeleceng.2017.09.018).

[26] LI B, REN W, FU D, et al. Benchmarking single image dehazing and beyond[J]. IEEE Transactions on Image Processing, 2019, 28(1): 492-505. 